\title{
ON ABSOLUTE LOGARITHMIC SUMMABILITY OF A SEQUENCE RELATED TO A FOURIER SERIES
}

\author{
R. MOHANTY and B. MisRa
}

(Received January, 10, 1954)

1. Definition A. Let $\lambda(\omega)$ be continuous, differentiable and monotone increasing in $(A, \infty)$, where $A$ is some positive number, and let $\lambda(\omega) \rightarrow \infty$ as $\omega \rightarrow \infty$. Suppose $\Sigma u_{n}$ is a given infinite series and let

$$
c(\omega)=\sum_{n \leqq \omega}\{\lambda(\omega)-\lambda(n)\} u_{n} .
$$

The series $\Sigma u_{n}$ is said to be summable $|R, \lambda(n), 1|$ if

$$
\int_{A}^{\infty}\left|d\left[\frac{c(\omega)}{\lambda(\omega)}\right]\right|<\infty,
$$

i. e. if

$$
\int_{A}^{\infty} \frac{\lambda^{\prime}(\omega)}{\{\lambda(\omega)\}^{2}}\left|\sum_{n \leqq \omega} \lambda(n) u_{n}\right| d \omega<\infty .
$$

Definition B. Suppose $\left\{t_{n}\right\}$ is a given sequence and let $\tau_{n}=\left(t_{1}+\frac{1}{2} t_{2}+\right.$ $\left.\cdots+\frac{1}{n} t_{n}\right) / \log n$. If $\tau_{n} \rightarrow t$ as $n \rightarrow \infty$, then the sequence $\left\{t_{n}\right\}$ is said to be summable $(R, \log n, 1)$ to $t$. If the sequence $\left\{\tau_{n}\right\}$ is of bounded variation, i.e. ,if $\sum^{\infty}\left|\tau_{n}-\tau_{n+1}\right|<\infty$, the sequence is said to be summable $|R, \log n, 1|$.

2. Let $\varphi(t)$ be an even function integrable in the sense of Lebesgue in $(0, \pi)$ and defined outside $(-\pi, \pi)$ by periodicity. We assume that the constant term in the Fourier series of $\varphi(t)$ is zero and that the special point to be considered is the origin. In these circumstances

where

$$
\varphi(t) \sim \sum_{1}^{\infty} a_{n} \cos n t
$$

$$
a_{n}=\frac{2}{\pi} \int_{0}^{\pi} \phi(t) \cos n t d t,
$$

and we are to consider the series $\sum_{1}^{\infty} a_{n}$. It is well-known that these formal simplifications do not limpair the generality of the problem. We write $s_{*}$ for $\sum_{1}^{n} a_{k}$ and use the following notations. 


$$
\begin{array}{ll}
\Phi_{\alpha}(t)=\frac{1}{\Gamma(\alpha)} \int_{0}^{t}(t-u)^{\alpha-1} \varphi(u) d u(t>0) \text { and } & (0<\alpha<1), \\
\Phi_{0}(t)=\varphi(t), & (0 \leqq \alpha<1), \\
\varphi_{\alpha}(t)=\Gamma(\alpha+1) t^{-\alpha} \Phi_{\alpha}(t) & (\Delta>0), \\
p(\omega)=\sum_{n \leqq \omega} e^{(\log n)^{\Delta}(\log n)^{-1} a_{n}} & \\
\xi(\omega, t)=\sum_{n \leqq \omega} e^{(\log n)^{\Delta}}(\log n)^{-1} \cos n t, & (0 \leqq u \leqq \pi), \\
\eta(\omega, t)=\sum_{n \leqq \omega} e^{(\log n)}(\log n)^{-1} n^{-1} \sin n t, & \\
g(\omega, u)=\frac{1}{\Gamma(1-\alpha)} \int_{u}^{\pi}(t-u)^{-\alpha} \xi(\omega, t) d t & (0 \leqq u \leqq \pi) . \\
G(\omega, u)=\frac{1}{\Gamma(\alpha+1)} \int_{0}^{u} v^{\alpha} \frac{d}{d v} g(\omega, v) d v &
\end{array}
$$

3. The following result is well-known:

(a) If $\phi(t)=o(1)$, then $s_{n}=o(\log n)$.

The statement (a) is equivalent to

(b) If $\varphi(t)=o(1)$, then the sequence $\left\{n a_{n}\right\}$ is summable $(R, \log n, 1)$ to 0 .

It is reasonable to expect that the analoge of (b) for absolute summability would be

(c) If $\phi(t)$ is of bounded variation, the sequence $\left\{n a_{n}\right\}$ is summable $|R, \log n, 1|$.

We shall however show that the statement (c) is false. We first prove the following

LEmMA. If the series $\Sigma u_{n}$ is summable $|R, \log n, 1|$, the necessary and sufficient condition that it is absolutely convergent is that the sequence $\left\{(n \log n) u_{n}\right\}$ is summable $|R, \log n, 1|$.

Proof of Lemma. We have on writing $\sigma_{n}=\sum_{1}^{n} u_{k} \log k$, the identity

$$
\frac{\sigma_{n}}{\log n}-\frac{\sigma_{n+1}}{\log (n+1)}=\sigma_{n} \frac{\log \left(1+\frac{1}{n}\right)}{\log n \log (n+1)}-u_{n+1}, \text { for } n \geqq 2 .
$$

Hence we have the following inequalities

$$
\sum_{2}^{\infty}\left|\frac{\sigma_{n}}{\log n}-\frac{\sigma_{n+1}}{\log (n+1)}\right|<A \sum_{2}^{\infty} \frac{\left|\sigma_{n}\right|}{n(\log n)^{2}}+\sum_{2}^{\infty}\left|u_{n+1}\right|
$$

and 


$$
\sum_{2}^{\infty}\left|u_{n+1}\right|<A \sum_{2}^{\infty} \frac{\left|\sigma_{n}\right|}{n(\log n)^{2}}+\sum_{2}^{\infty}\left|\frac{\sigma_{n}}{\log n}-\frac{\sigma_{n+1}}{\log (n+1)}\right| .
$$

Since the series $\Sigma u_{n}$ is summable $|R, \log n, 1|$, we have, by Definition A,

$$
\int_{2}^{\infty} \frac{1}{\omega(\log \omega)^{2}}\left|\sum_{n \leqq \omega} \log n u_{n}\right| d \omega<\infty
$$

from which it follows that

$$
\sum_{2}^{\infty} \frac{\left|\sigma_{n}\right|}{n(\log n)^{2}}<\infty
$$

The proof of the Lemma then follows from Definition B and (3.2), (3.3) and (3.5).

In order to prove that the statement (c) is false we observe that the series $\sum_{2}^{\infty} a_{n} / \log n$ is summable $|R, \log n, 1|$ if

$$
\int_{2}^{\infty} \frac{\left|s_{[\omega]}\right|}{\omega(\log \omega)^{2}} d \omega<\infty
$$

The above condition is obviously satisfied when $\varphi(t)$ is of bounded variation in $(0, \pi)$ and indeed when a continuity condition of the type $\phi(t)=$ $O\left\{\left(\log \frac{1}{t}\right)^{-\eta}\right\}(0<\eta<1)$ is satisfied; since with the latter condition we can assert that $s_{n}=O\left\{(\log n)^{1-\eta}\right\}^{1)}$. But bounded variation of $\varphi(t)$ in $(0, \pi)$ is not sufficient to ensure absolute convergence of the series $\sum_{2}^{\infty} a_{n} / \log n$. [3] Hence writing $a_{n} / \log n$ for $u_{n}$ in the Lemma proved above, we can easily see that bounded variation of $\phi(t)$ alone is not sufficient ensure summability $|R, \log n, 1|$ of the sequence $\left\{n a_{n}\right\}$ :

4. We now proceed to establish some tests for the absolute convergence of the series $\sum_{2}^{\infty} a_{n} / \log n$. In the first instance we prove the

THEOREM. If $\varphi_{a}(t)$ is of bounded variation in $(0, \pi)$, then the series $\sum_{2}^{\infty} a_{n} / \log n$ is summable $\left|R, e^{(\log n)^{\Delta}} ; 1\right|$, where

$$
0<\alpha<1 \text { and } \Delta=1+\frac{1}{\alpha} \text {. }
$$

We first establish the following inequalities :

1) This can be proved with a slight modification of the proof of the theorem " $s_{n}=o(\log n)$, when $\phi(t)=o(1) "$ as given in Titchmarsh [4]. 


$$
\begin{aligned}
& \xi(\omega, t)=O\left\{e^{(\log \omega) \Delta} \omega(\log \omega)^{-\Delta}\right\} \\
& \xi(\omega, t)=O \cdot\left\{e^{(\log \omega) \Delta} t^{-1}(\log \omega)^{-1}\right\} \\
& \eta(\omega, t)=O\left\{e^{(\log \omega) \Delta}(\log \omega)^{-\Delta}\right\} \\
& \eta(\omega, t)=O\left\{e^{(\log \omega) \Delta} t^{-1}(\omega \log \omega)^{-1}\right\} \\
& g(\omega, u)=O\left\{e^{\left.(\log \omega) \Delta \omega^{\alpha}(\log \omega)^{-\Delta}\right\}}\right. \\
& g(\omega, u)=O\left\{e^{(\log \omega)^{\Delta} \omega^{-1+\alpha} u^{-1}(\log \omega)^{-1}}\right\} \\
& G(\omega, u)=O\left\{e^{\left.(\log \omega)^{\Delta} \omega^{\alpha} u^{\alpha}(\log \omega)^{-\Delta}\right\}}\right. \\
& G(\omega, u)=O\left\{e^{(\log \omega)} \omega^{-1+\alpha} u^{-1+\alpha}(\log \omega)^{-1}\right\}
\end{aligned}
$$

The inequalities (4.1) and (4.3) can be proved exactly in the same manner as in [3]. The inequalities (4.2) and (4.4) can be proved by using Abel's Lemma.

PROOF OF (4.5) AND (4.6). For $u+\omega^{-1}<\pi^{2)}$, we write

$$
\begin{aligned}
& \Gamma(1-\alpha) g(\omega, u)=\int_{u}^{u+\omega^{-1}}+\int_{u+\omega}^{\pi}=I_{1}+I_{2} \\
&\left|I_{1}\right|<A e^{(\log \omega)^{\Delta}} \int_{u}^{u+\omega^{-1}}(t-u)^{-\alpha} \min \left[\omega(\log \omega)^{-\Delta}, t^{-1}(\log \omega)^{-1}\right] d t \\
&<A e^{\log \omega)^{\Delta}} \min \left[\omega(\log \omega)^{-\Delta}, u^{-1}(\log \omega)^{-1}\right] \int_{u}^{u+\omega-1}(t-u)^{-\alpha} d t \\
&=O\left\{e^{\left.(\log \omega)^{\Delta} \omega^{-1+\alpha}\right\} \min \left[\omega(\log \omega)^{-\Delta}, u^{-1}(\log \omega)^{-1}\right]}\right. \\
& I_{2}=\left(\frac{1}{\omega}\right)^{-\alpha} \int_{u+\omega^{-1}}^{\rho} \xi(\omega, t) d t \\
&=\omega^{\alpha}[\eta(\omega, t)]_{u+\omega^{-1}}^{p}\left(u+\omega^{-1}<\rho<\pi\right) \\
&=O\left\{e^{(\log \omega) \Delta} \omega^{-1+\alpha}\right\} \min \left[\omega(\log \omega)^{-\Delta}, u^{-1}(\log \omega)^{-1}\right]
\end{aligned}
$$

PROOF of (4.7). We have

$$
\begin{aligned}
\Gamma(\alpha+1) G(\omega, u) & =\int_{v}^{u} v^{\alpha} \frac{d}{d v} g(\omega, v) d v \\
& =u^{\alpha} g(\omega, u)-\alpha \int_{0}^{u} v^{\alpha-1} g(\omega, v) d v \\
& =O\left\{e^{\left(\log \omega^{\prime} \Delta\right.} \omega^{\alpha} u^{\alpha}(\log \omega)^{-\Delta}\right\}, \text { by (4.6). }
\end{aligned}
$$

ProOF of (4. 8). It is easy to see, that

Further

$$
g(\omega, \pi)=O\left\{e^{(\log \omega)^{\Delta}} \omega^{-1+\alpha}(\log \omega)^{-1}\right\} .
$$

$$
\Gamma(\alpha+1) G(\omega, \pi)=\left[v^{\alpha} g(\omega, v)\right]_{0}^{\pi_{3}}-\alpha \int_{0}^{\pi} v^{\alpha-1} g(\omega, v) d v
$$

But

2) For $u+\omega^{-1} \geqq \pi$, the integral need not be split up and the arguments for $I_{1}$ will hold for the integral. 


$$
\begin{aligned}
\Gamma(1-\alpha) & \int_{0}^{\pi} v^{\alpha-1} g(\omega, v) d v=\int_{0}^{\pi} v^{\alpha-1}\left(\int_{v}^{\tau}(t-v)^{-\alpha} \xi(\omega, t) d t\right) d v \\
& =\int_{0}^{\pi} \xi(\omega, t)\left(\int_{0}^{t} v^{\alpha-1}(t-v)^{-\alpha} d v\right) d t \\
& =\int_{0}^{\pi} \xi(\omega, t)\left(\int_{0}^{1} x^{\alpha-1}(1-x)^{-\alpha} d x\right) d t \\
& =0 .
\end{aligned}
$$

Hence

(4. 10) $\quad G(\omega, \pi)=O\left\{e^{(\log \omega)^{\Delta}} \omega^{-1+\alpha}(\log \omega)^{-1}\right\}$, by (4.9).

To prove (4.8), we have

$$
\begin{aligned}
& \Gamma(\alpha+1)\{G(\omega, \pi)-G(\omega, u)\}=\left[v^{\alpha} g(\omega, v)\right]_{u}^{\pi}-\alpha \int_{u}^{\pi} v^{\alpha-1} g(\omega, v) d v \\
& =O\left\{e^{(\log \omega)^{\Delta} \omega^{-1+\alpha}(\log \omega)^{-1}}\right\}+O\left\{e^{(\log \omega)^{\Delta} \omega^{-1+\alpha} u^{-1+\alpha}(\log \omega)^{-1}}\right\} \\
& -\alpha \int_{u}^{\pi} v^{\alpha-1} O\left\{e^{(\log \omega)^{\Delta} \omega^{-1+\alpha} v^{-1}(\log \omega)^{-1}}\right\} d v .
\end{aligned}
$$

Since $\alpha<1$, using (4.10)

$$
G(\omega, u)=O\left\{e^{(\log \omega)^{\Delta}} \omega^{-1+\alpha} u_{-1+\alpha}^{-1}(\log \omega)^{-1}\right\}
$$

Proof of Theorem. To prove the theorem, we have to show that when $\Delta=1+\frac{1}{\alpha}$

$$
I=\int_{2}^{\infty} \Delta \omega^{-1}(\log \omega)^{\Delta-1} e^{-(\log \omega) \Delta}|p(\omega)| d \omega<\infty .
$$

We have

Hence

$$
\begin{aligned}
a_{n} & =\frac{2}{\pi} \int_{0}^{\pi} \varphi(t) \cos n t d t \\
& =\frac{2}{\pi} \cdot \frac{1}{\Gamma(1-\alpha)} \int_{0}^{\pi} \cos n t \int_{0}^{t}(t-u)^{-\alpha} d \Phi_{\alpha}(u) \\
& =\frac{2}{\pi} \cdot \frac{1}{\Gamma(1-\alpha)} \int_{0}^{\pi} d \Phi_{\alpha}(u) \int_{u}^{\pi}(t-u)^{-\alpha} \cos n t d t,[1]
\end{aligned}
$$

$$
\begin{aligned}
\frac{1}{2} \pi p(\omega) & =\frac{1}{\Gamma(1-\alpha)} \int_{0}^{\pi} d \Phi_{\alpha}(u) \int_{u}^{\pi}(t-u)^{-\alpha} \sum_{n \leqq \omega} e^{(\log n)^{\Delta}}(\log n)^{-1} \cos n t d t \\
& =\frac{1}{\Gamma(1-\alpha)} \int_{0}^{\pi} d \Phi_{\alpha}(u) \int_{u}^{\pi}(t-u)^{-\alpha} \xi(\omega, t) d t
\end{aligned}
$$




$$
\begin{aligned}
& =\int_{0}^{\pi} g(\omega, u) d \Phi_{\alpha}(u) \\
& =\left[g(\omega, u) \Phi_{\alpha}(u)\right]_{0}^{\pi}-\int_{0}^{\pi} \Phi_{\alpha}(u) \frac{d}{d u} g(\omega, u) d u .
\end{aligned}
$$

Further since $\varphi_{a}(+0)$ is finite

$$
\begin{aligned}
& \int_{0}^{\pi} \Phi_{\alpha}(u) \frac{d}{d u} g(\omega, u) d u=\frac{1}{\Gamma(\alpha+1)} \int_{0}^{\pi} \phi_{\alpha}(u) u^{\alpha} \frac{d}{d u} g(\omega, u) d u \\
= & \left.\frac{1}{\Gamma(\alpha+1)}\left[\phi_{\alpha}(u) \int_{0}^{u} v^{\alpha} \frac{d}{d v} g(\omega, v) d v\right]_{0}^{\pi}-\frac{1}{\Gamma(\alpha+1)} \int_{0}^{\pi} d \phi_{\alpha}(u)\right] \int_{0}^{u} v^{\alpha} \frac{d}{d v} g(\omega, v) d v \\
= & \phi_{\alpha}(\pi) G(\omega, \pi)-\int_{0}^{\pi} G(\omega, u) d \phi_{\alpha}(u) .
\end{aligned}
$$

So we have finally

$$
\begin{aligned}
\frac{1}{2} \pi p(\omega) & =\left[g(\omega, u) \Phi_{\alpha}(u)\right]_{0}^{\pi}-\phi_{\alpha}(\pi) G(\omega, \pi)+\int_{0}^{\pi} G(\omega, u)^{\prime} d \varphi_{\alpha}(u) \\
& =O\left\{e^{\left.(1 \log \omega)^{\Delta} \omega^{-1+\alpha}(\log \omega)^{-1}\right\}+\int_{0}^{\pi} G(\omega, u) d \phi_{\alpha}(u), \text { by }(4.9) \text { and }(4.10)}\right.
\end{aligned}
$$

Hence

$$
I<A \int_{2}^{\infty} \frac{(\log \omega)^{\Delta-2}}{\omega^{2-\alpha}} d \omega+\frac{2}{\pi} \int_{2}^{\infty} \frac{\Delta}{\omega}(\log \omega)^{\Delta-1} e^{-(\log \omega)}\left|\int_{0}^{\pi} G(\omega, u) d \varphi_{\alpha}(u)\right| d \omega
$$

The integral

and the integral

$$
\int_{2}^{\infty} \frac{(\log \omega)^{\Delta-2}}{\omega^{2-\alpha}} d \omega<\infty
$$

$$
\begin{aligned}
& \int_{2}^{\infty} \frac{\Delta}{\omega}(\log \omega)^{\Delta-1} e^{(\log \omega)^{\Delta}}\left|\int_{0}^{\pi} G(\omega, u) d \phi_{\alpha}(u)\right| d \omega \\
\leqq & \int_{0}^{\pi}\left|d \varphi_{\alpha}(u)\right| \int_{2}^{\infty} \Delta \omega^{-1}(\log \omega)^{\Delta-1} e^{-(\log \omega)^{\Delta}}|G(\omega, u)| d \omega
\end{aligned}
$$

Since $\varphi_{a}(t)$ is of bounded variation in $(0, \pi)$, to prove the theorem it will be sufficient to show that

$$
J=\int^{\infty} \Delta \omega^{-1}(\log \omega)^{\Delta-1} e^{-(\log \omega)^{\Delta}}|G(\omega, u)| d \omega<\infty .
$$

Writing

$$
J=\int_{2}^{\tau}+\int_{\tau}^{\infty}=J_{1}+J_{2}, \quad \text { where } \tau=\frac{k}{u}\left(\log \frac{k}{u}\right)^{\frac{1}{\alpha}}, \quad(k>e \pi),
$$


we have

$$
\begin{aligned}
J_{1} & =\int_{2}^{\tau} \Delta \omega^{-1}(\log \omega)^{\Delta-1} e^{-(\log \omega)^{\Delta}} O\left\{e(\log \omega)^{\Delta} \omega^{\alpha} u^{\alpha}(\log \omega)^{-\Delta}\right\} d \omega, \text { by (4.7) } \\
& =O\left\{u^{\alpha} \int_{2}^{\tau} \frac{\omega^{-1+\alpha}}{\log \omega} d \omega\right\}=O(1),
\end{aligned}
$$

and

$$
\begin{aligned}
& 2=\int_{\tau}^{\infty} \Delta \omega^{-1}(\log \omega)^{-1} e^{-(\log \omega)^{\Delta}} O\left\{e^{(\log \omega)^{\Delta} \omega^{-1+\alpha} u^{-1+\alpha}(\log \omega)^{-1}}\right\} d \omega, \text { by } \\
& =O\left\{u^{-1+\alpha} \int_{\tau}^{\infty} \frac{(\log \omega)^{\Delta-2}}{\omega^{2-\alpha}} d \omega\right\}=O(1), \text { if } \Delta=1+\frac{1}{\alpha} .
\end{aligned}
$$

Hence the theorem is proved.

5. The following theorems have been proved elsewhere [2], [3].

THEOREM $A$. If (i) the sequence $\left\{\frac{u_{n} \lambda(n)}{\lambda(n)-\lambda(n-1)}\right\}$ is of bounded variation, (ii) the sequence $\left\{\frac{\lambda(n)}{\lambda(n+1)}\right\}$ is of bounded variation and (iii) the series $\Sigma u_{n}$ is summable $|R, \lambda(n), 1|$, then the series is absolutely convergent.

THEOREM $B$. If $\varphi(t)$ is of bounded variation in $(0, \pi)$ then the series $\Sigma a_{n} / \log n$ is summable $\left|R, e^{n^{\alpha}}, 1\right|$, where $0<\alpha<1$.

Combining theorems $\mathrm{A}$ and $\mathrm{B}$ on the one hand and Theorem $\mathrm{A}$ and the Theorem proved above on the other, we have the following criteria for the absolute convergence of the series $\Sigma a_{n} / \log n$.

(I) If $\phi(t)$ is of bounded variation in $(0, \pi)$ and the seqnence $\left\{n^{\delta}(\log n)^{-1} a_{n}\right\}$ is of bounded variation for $\delta>0$ then the series $\Sigma a_{n} / \log n$ is absolutely convergent.

(II) If $\phi_{\alpha}(t)$ is of bounded variation in $(0, \pi)$ for $0<\alpha<1$ and the sequence $\left\{\frac{n}{(\log n)^{1+\frac{1}{\alpha}}} a_{n}\right\}$ is of bounded variation, then the series $\Sigma a_{n} / \log n$ is absolutely convergent.

We have already remarked that if $\varphi(t)=O\left\{\left(\log \frac{1}{t}\right)^{-\eta}\right\}(0<\eta<1)$, then the series $\Sigma a_{n} / \log n$ is summable $|R, \log n, 1|$. Combining this with Theorem A, we have

(III) If $\phi(t)=O\left\{\left(\log \frac{1}{t}\right)^{-\eta}\right\}(0<\eta<1)$ and the sequence $\left\{n a_{n}\right\}$ is of bounded variation, then the series $\Sigma a_{n} / \log n$ is absolutely convergent.

Reverting to the original problem ot summability $|R, \log n, 1|$ of the sequence $\left\{n a_{n}\right\}$, we have the following results, which by virtue of the Lemma of this paper, which by victure of the lemma of this paper are practically restatements of (I)-(III) above. 
(d) If $\varphi^{\prime}(t)$ is of bounded variation in $(0, \pi)$ and the sequence $\left\{n^{\delta}(\log n)^{-1} a_{n}\right\}$ is of bounded variation for $\delta>0$, then the sequence $\left\{n a_{n}\right\}$ is summable $|R, \log n, 1|$ :

(e) If $\varphi_{\alpha}(t)$ is of bounded variation in $(0, \pi)$ for $0<\alpha<1$ and the sequence $\left\{\frac{n a_{n}}{(\log n)^{1+\frac{1}{\alpha}}}\right\}$ is of bounded variation, then the sequence $\left\{n a_{n}\right\}$ is summable $|R, \log n, 1|$; and

(f) If $\phi(t)=O\left\{\left(\log \frac{1}{t}\right)^{-\eta}\right\}(0<\eta<1)$ and the sequence $\left\{n a_{n}\right\}$ is of bounded variation, then the sequence is summable $|R, \log n, 1|$.

\section{REFERENCES}

[1] Bosanquet, L.S. The absolute Cesàro summability of a Fourier series, Proceedings of the London Mathematical Society, Ser. 2, vol. 41, (1936), 517-528.

[2] MOHANTY, R. A criterion for the absolute covergence of a Fourier series, Proceedings of the London Mathematical society, ser.2, vol. 51, (1949), 186-196.

[3] Mohanty, $\mathbf{R}$. On the absolute Riesz summability 'of a Fourier series and allied series, Proceedings of the London Mathematical society, ser.2, vol. 52, (1951), 295-320.

[4] Titchmarsh, E. C. Theory of Functions (1939), Oxford University Press.

Department of Mathematics, Ravenshaw College, Cuttak, India. 